\title{
Three-Dimensional Printing Fully Biobased Heat-Resistant Photoactive Acrylates from Aliphatic Biomass
}

Jia-Tao Miao, ${ }^{\dagger}$ Shuqiang Peng, ${ }^{\dagger,+}$ Meiying Ge, ${ }^{\dagger}$ Yuewei Li, ${ }^{\dagger},+$ Jie Zhong, ${ }^{\dagger}$ Zixiang Weng, ${ }^{\dagger}$ Lixin $W u,{ }^{*}+$ and Longhui Zheng ${ }^{*}+$

$\dagger$ CAS Key Laboratory of Design and Assembly of Functional Nanostructures, Fujian Key Laboratory of Nanomaterials, Fujian Institute of Research on the Structure of Matter, Chinese Academy of Sciences, Fuzhou 350002, People’s Republic of China.

$¥$ University of Chinese Academy of Sciences, Beijing 100049, People’s Republic of China

Corresponding Authors

*E-mail: lxwu@fjirsm.ac.cn (L.W.)

*E-mail: lhzheng@fjirsm.ac.cn (L.Z.)

Supporting Information contains 8 pages including 7 Figures and 1 Table. 


\section{Index}

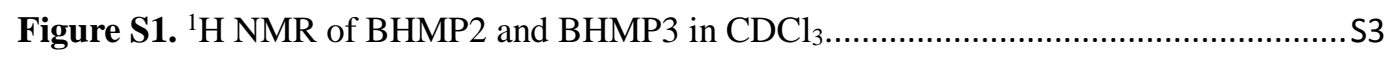

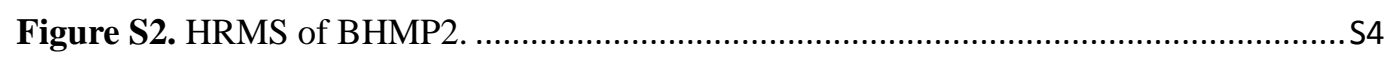

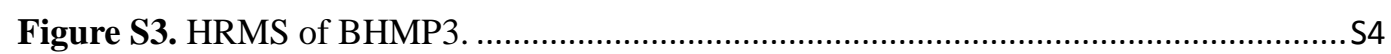

Figure S4. FTIR spectra of BHMP2 and BHMP3 resins before and after UV curing. ..........S5

Figure S5. PALS spectra of BHMP2 and BHMP3 resins................................................ 5

Figure S6. (a) 3D objects printed by BHMP3 resin with $100 \mathrm{~g}$ weight on it after insulation at $250{ }^{\circ} \mathrm{C}$ for $1 \mathrm{~h}$. (b) 3D objects printed by BHMP3 resin with $1000 \mathrm{~g}$ weight on it after insulation at $180^{\circ} \mathrm{C}$ for $1 \mathrm{~h}$. .56

Figure S7. 3D objects printed by BHMP2 resin with $100 \mathrm{~g}$ (a) or $1000 \mathrm{~g}$ (b) weight on it broke down after heating in an oven. .56

Table S1. A comparison of bio-degree and $T_{\mathrm{g}}$ of biobased and petroleum-based acrylates for $3 \mathrm{D}$ printing in literature .S7 


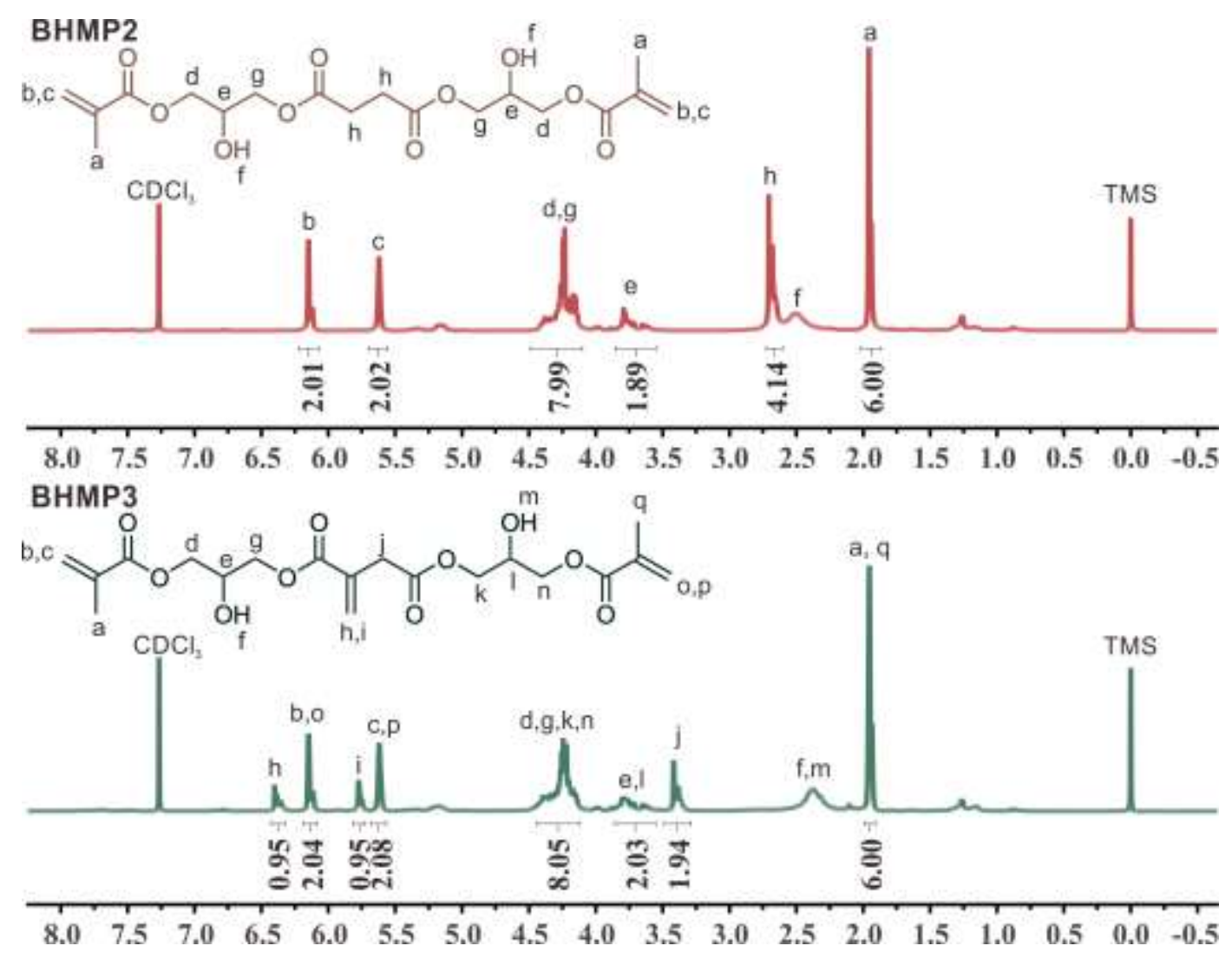

Figure S1. ${ }^{1} \mathrm{H}$ NMR of BHMP2 and BHMP3 in $\mathrm{CDCl}_{3}$. 


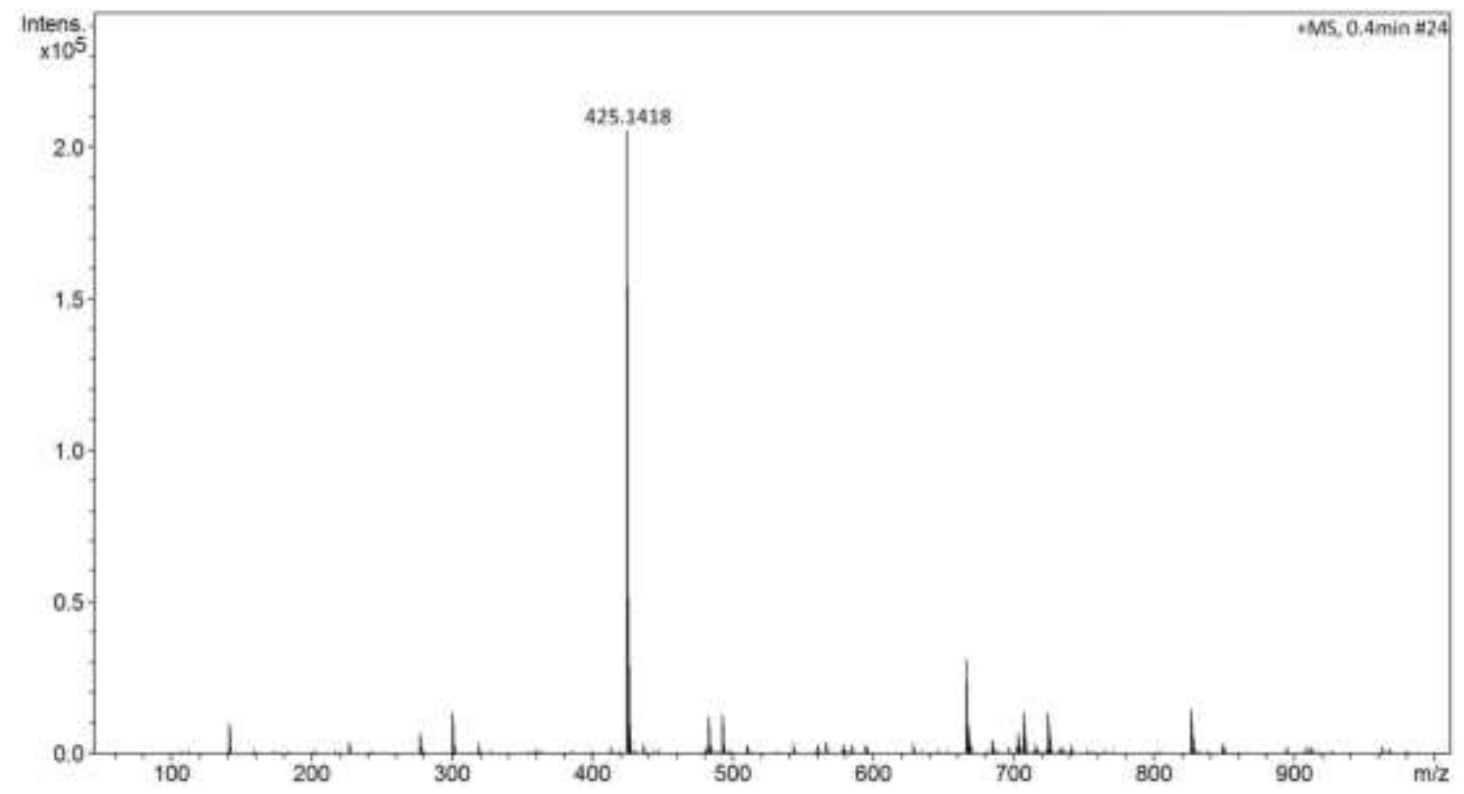

Figure S2. HRMS of BHMP2.

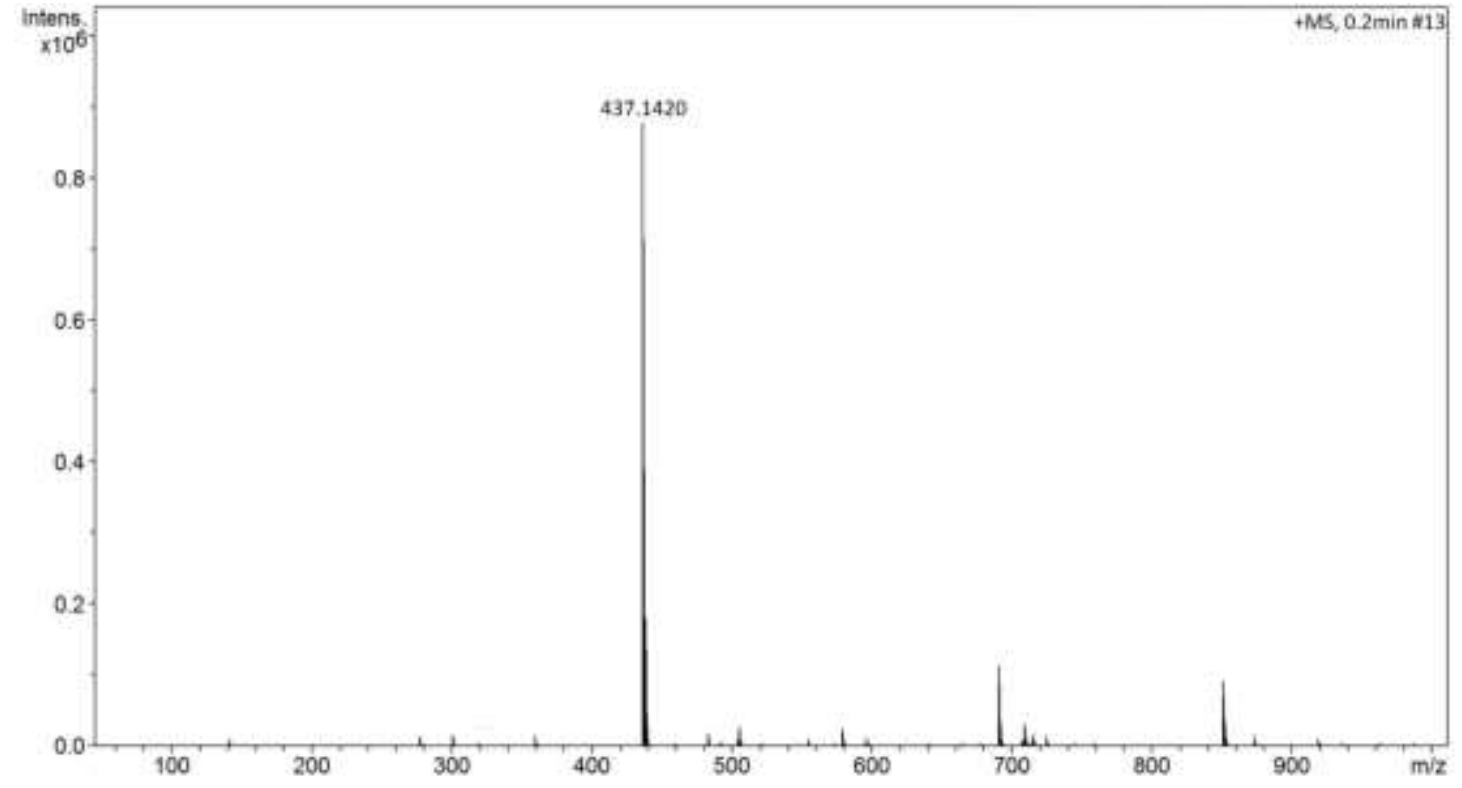

Figure S3. HRMS of BHMP3. 


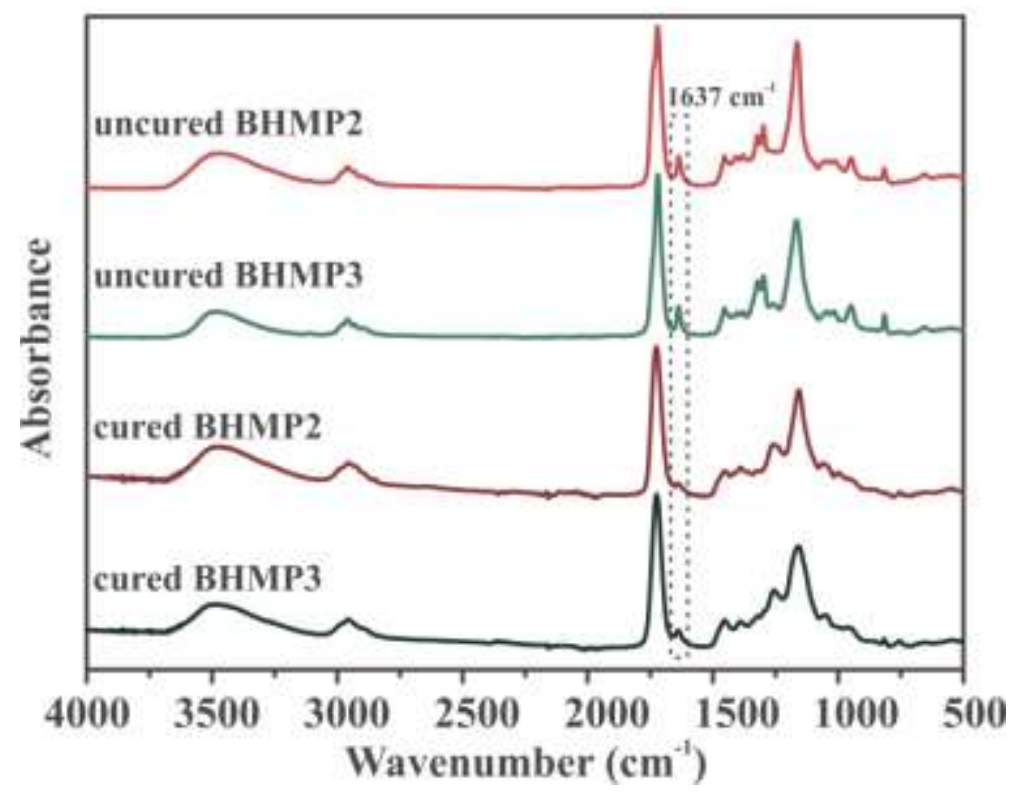

Figure S4. FTIR spectra of BHMP2 and BHMP3 resins before and after UV curing.

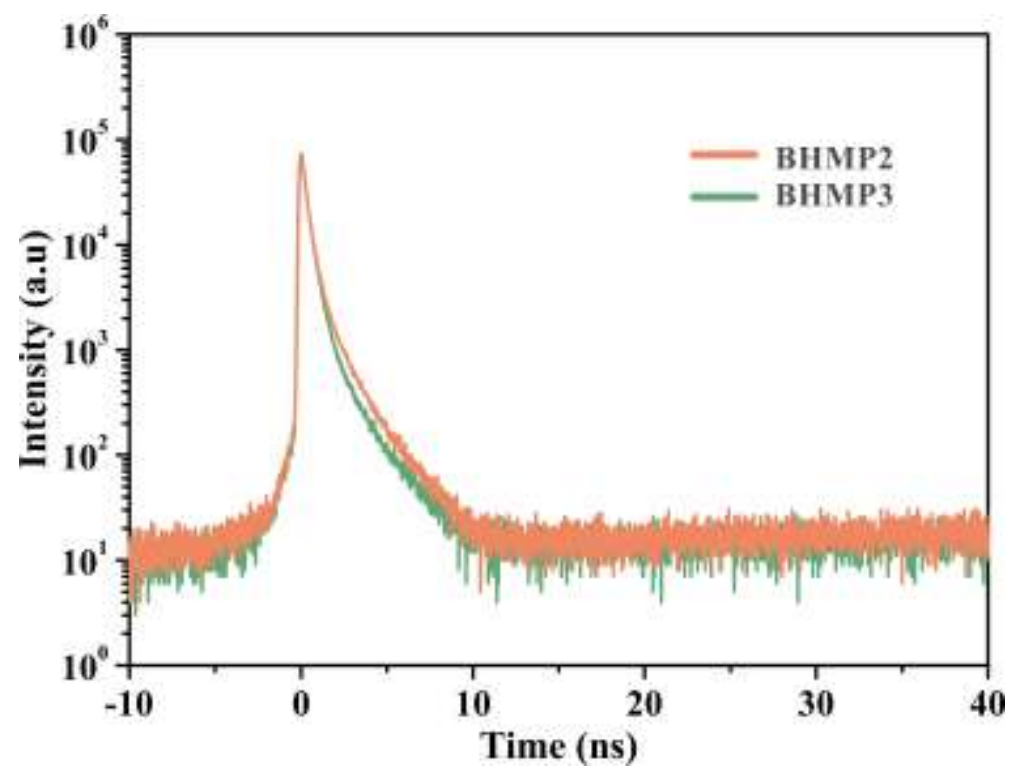

Figure S5. PALS spectra of BHMP2 and BHMP 3 resins. 


\section{Supporting Information}

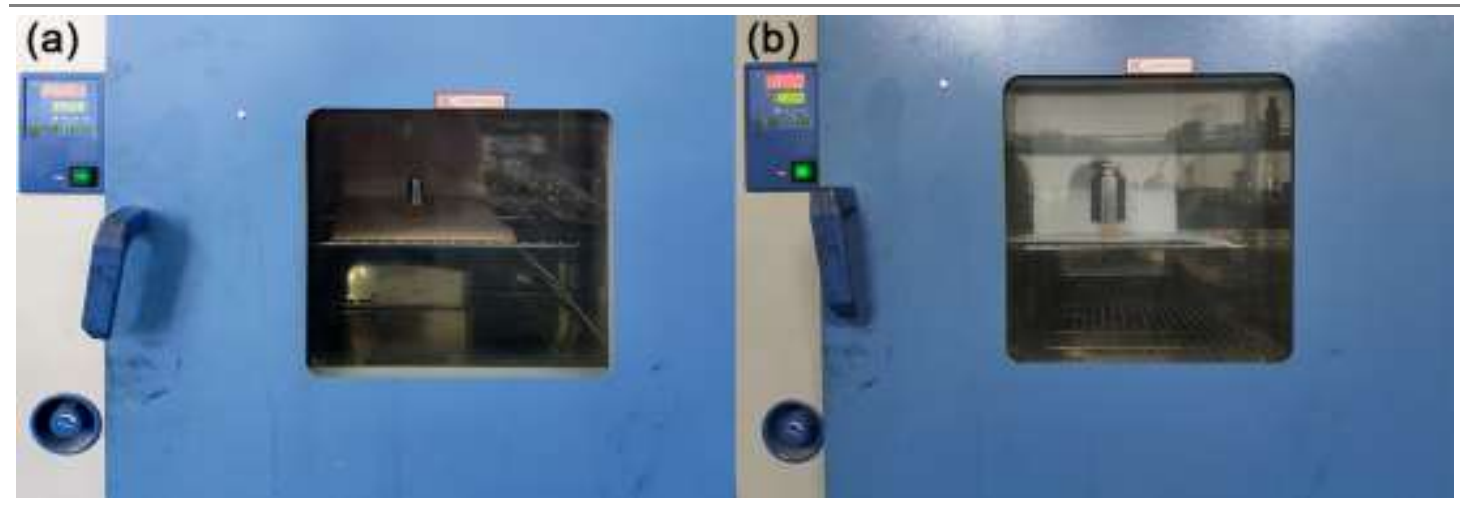

Figure S6. (a) 3D objects printed by BHMP3 resin with $100 \mathrm{~g}$ weight on it after insulation at $250{ }^{\circ} \mathrm{C}$ for $1 \mathrm{~h}$. (b) $3 \mathrm{D}$ objects printed by BHMP3 resin with $1000 \mathrm{~g}$ weight on it after insulation at $180{ }^{\circ} \mathrm{C}$ for $1 \mathrm{~h}$.

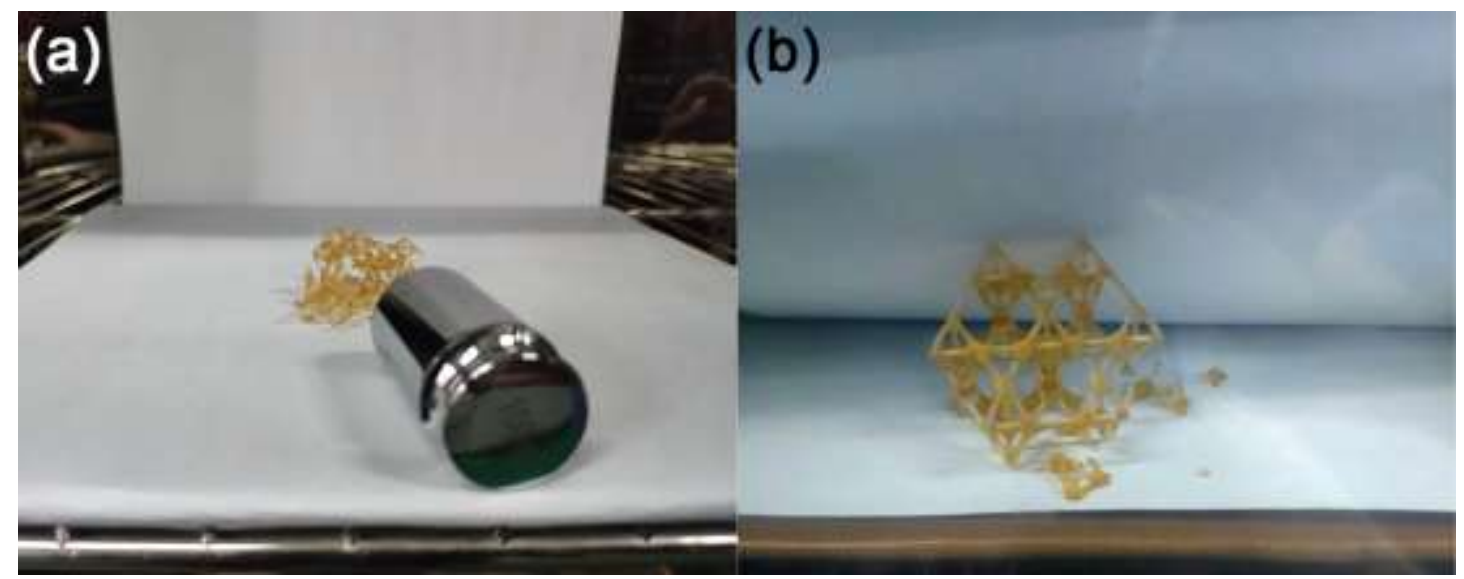

Figure S7. 3D objects printed by BHMP2 resin with $100 \mathrm{~g}$ (a) or $1000 \mathrm{~g}$ (b) weight on it broke down after heating in an oven. 


\section{Supporting Information}

Table S1. A comparison of bio-degree and $T_{\mathrm{g}}$ of biobased and petroleum-based acrylates for 3D printing in literature.

\begin{tabular}{|c|c|c|c|}
\hline Acrylate resin & Bio-degree $^{\text {a) }}$ & $T_{g}\left({ }^{\circ} \mathrm{C}\right)^{\mathrm{b})}$ & Ref \\
\hline BHMP2 & Fully & 147 & \multirow{2}{*}{ This work } \\
\hline BHMP3 & Fully & 183 & \\
\hline $\mathrm{pE}$ & Partially & 45.0 & \multirow{4}{*}{1} \\
\hline pGE 75-25 & Partially & 79.7 & \\
\hline pGET 60-20-20 & Partially & 130.9 & \\
\hline pGEV 60-20-20 & Partially & 107.5 & \\
\hline BMPR-06 & Partially & 80.2 & \multirow{3}{*}{2} \\
\hline BMPR-09 & Partially & 96.9 & \\
\hline BMPR-10 & Partially & 78.1 & \\
\hline 3DPT-2-8h & Partially & $\approx 51$ & \multirow{2}{*}{3} \\
\hline 3DPT-3-8h & Partially & $\approx 63$ & \\
\hline LR5 & Partially & - & \multirow{3}{*}{4} \\
\hline LR10 & Partially & - & \\
\hline LR15 & Partially & - & \\
\hline AWSO & Partially & -10 & 5 \\
\hline soy & Partially & $20^{c)}$ & 6 \\
\hline SLR & None & 76.8 & 7 \\
\hline 3DPRTs & None & $\approx 55$ & 8 \\
\hline AA-BPA-SMP & None & 71 & 9 \\
\hline BMA-PEGDMA & None & 60 & 10 \\
\hline $10 \mathrm{wt} \%$ HDDA & None & 48.7 & \multirow{5}{*}{11} \\
\hline 20wt $\%$ HDDA & None & 52.9 & \\
\hline 30wt $\%$ HDDA & None & 58.8 & \\
\hline 40wt $\%$ HDDA & None & 67.6 & \\
\hline 50wt $\%$ HDDA & None & 76.3 & \\
\hline Polyimide1 & None & $\approx 380$ & 12 \\
\hline Polyimide2 & None & 242 & 13 \\
\hline
\end{tabular}

a) Biobased content is assigned as the "amount of biobased carbon in the material or product as a percent of the weight (mass) of the total organic carbon in the product" by the United States Department of Agriculture (USDA).

Fully: Fully biobased, biobased content $=100 \%$

Partially: Partially biobased, $0<$ biobased content $<100 \%$

None: Petroleum-based, biobased content $=0$

b) $T_{g}$ is determined as the peak of $\tan \delta$ curve from DMA.

c) $T_{g}$ is determined by DSC.

The symbol ' $\approx$ ' is used to indicate that the datum is an approximate value. 


\section{References}

(1) Ding, R.; Du, Y.; Goncalves, R. B.; Francis, L. F.; Reineke, T. M. Sustainable near UV-Curable Acrylates Based on Natural Phenolics for Stereolithography 3D Printing. Polym. Chem. 2019, 10 (9), 1067-1077.

(2) Guit, J.; Tavares, M. B. L.; Hul, J.; Ye, C.; Loos, K.; Jager, J.; Folkersma, R.; Voet, V. S. D. Photopolymer Resins with Biobased Methacrylates Based on Soybean Oil for Stereolithography. ACS Appl. Polym. Mater. 2020, 2 (2), 949-957.

(3) Lu, C.; Wang, C.; Yu, J.; Wang, J.; Chu, F. Two-Step 3D-Printing Approach toward Sustainable, Repairable, Fluorescent Shape-Memory Thermosets Derived from Cellulose and Rosin. ChemSusChem 2020, 13 (5), 893-902.

(4) Sutton, J. T.; Rajan, K.; Harper, D. P.; Chmely, S. C. Lignin-Containing Photoactive Resins for 3D Printing by Stereolithography. ACS Appl. Mater. Interfaces 2018, 10 (42), 36456-36463.

(5) Wu, B.; Sufi, A.; Ghosh Biswas, R.; Hisatsune, A.; Moxley-Paquette, V.; Ning, P.; Soong, R.; Dicks, A. P.; Simpson, A. J. Direct Conversion of Mcdonald's Waste Cooking Oil into a Biodegradable High-Resolution 3D-Printing Resin. ACS Sustainable Chem. Eng. 2020, 8 (2), 1171-1177.

(6) Miao, S.; Zhu, W.; Castro, N. J.; Nowicki, M.; Zhou, X.; Cui, H.; Fisher, J. P.; Zhang, L. G. 4D Printing Smart Biomedical Scaffolds with Novel Soybean Oil Epoxidized Acrylate. Sci. Rep. 2016, 6, 27226.

(7) Li, Y.; Zhong, J.; Wu, L.; Weng, Z.; Zheng, L.; Peng, S.; Zhang, X. High Performance Poss Filled Nanocomposites Prepared via UV-Curing Based on 3D Stereolithography Printing. Compos. Part A-Appl. S. 2019, 117, 276-286.

(8) Zhang, B.; Kowsari, K.; Serjouei, A.; Dunn, M. L.; Ge, Q. Reprocessable Thermosets for Sustainable Three-Dimensional Printing. Nat. Commun. 2018, 9 (1), 1831.

(9) Yang, C.; Boorugu, M.; Dopp, A.; Ren, J.; Martin, R.; Han, D.; Choi, W.; Lee, H. 4D Printing Reconfigurable, Deployable and Mechanically Tunable Metamaterials. Mater. Horiz. 2019, 6 (6), 1244-1250.

(10) Zhang, B.; Zhang, W.; Zhang, Z.; Zhang, Y.-F.; Hingorani, H.; Liu, Z.; Liu, J.; Ge, Q. Self-Healing Four-Dimensional Printing with an Ultraviolet Curable Double-Network Shape Memory Polymer System. ACS Appl. Mater. Interfaces 2019, 11 (10), 10328-10336.

(11) Wu, H.; Chen, P.; Yan, C.; Cai, C.; Shi, Y. Four-Dimensional Printing of a Novel Acrylate-Based Shape Memory Polymer Using Digital Light Processing. Mater. Design. 2019, 171, 107704.

(12) Hegde, M.; Meenakshisundaram, V.; Chartrain, N.; Sekhar, S.; Tafti, D.; Williams, C. B.; Long, T. E. 3D Printing All-Aromatic Polyimides Using Mask-Projection Stereolithography: Processing the Nonprocessable. Adv. Mater. 2017, 29 (31), 1701240.

(13) Guo, Y.; Ji, Z.; Zhang, Y.; Wang, X.; Zhou, F. Solvent-Free and Photocurable Polyimide Inks for 3D Printing. J. Mater. Chem. A 2017, 5 (31), 16307-16314. 